\title{
Relative synthesis rate of individual muscle proteins: a new approach
}

\author{
V. V. A. M. Schreurs, H. A. Boekholt, R. E. Koopmanschap and W. van \\ Rotterdam
}

Department of Animal Physiology, Agricultural University, Haarweg 10, 6709 PJ, Wageningen, Netherlands

Received 7 January 1985; accepted 7 May 1985

Key-words: muscle proteins, actin, myosin, synthesis, SDS gel filtration

\section{Summary}

This paper describes the estimation of the relative synthesis rate of muscle proteins by means of a new approach. This approach is an obvious alternative for isolation procedures, with usually low protein recovery. Loss of protein material is avoided by taking advantage of separating the muscle proteins in a single step by SDS gel filtration. As a consequence an overall impression of the relative synthesis rate of the main muscle proteins can be visualized. This can be useful in comparative studies describing the influence of physiological parameters on the synthesis of individual muscle proteins.

\section{Introduction}

The estimation of the absolute synthesis rate of proteins is faced with several theoretical and practical pitfalls. In general, these have been discussed by Waterlow et al. (1978). If within a single tissue only the relative synthesis rate of individual proteins is studied most of these problems are less disturbing if they account to the same degree for all proteins involved.

The relative synthesis rate of proteins which share a common amino acid precursor pool is usually estimated by comparing the incorporation rate of a labelled precursor amino acid or more accurate the specific activity of that amino acid in the proteins.

Obviously, data for individual proteins can only be attained from purified fractions. A severe practical problem, discussed by Lobley \& Lovie (1979), is the possible change of the specific activity of a protein during the isolation procedure, due to an unequal loss of labelled and unlabelled material.

Loss of material, usually inherent to isolation procedures, is avoided if the proteins are separated in a single-step procedure. With that aim we have previously described a method for the separation of muscle proteins by means of gel filtration in 
the presence of the detergent sodium dodecyl sulphate (SDS) (Schreurs et al., 1983).

The present paper describes a new approach for the estimation and visualization of the relative synthesis rate of the main muscle proteins. The significance of this approach is discussed.

\section{Materials and methods}

\section{Labelling of muscle proteins}

Three New Zealand White rabbits $(\simeq 4 \mathrm{~kg})$ received a constant infusion of $50 \mu \mathrm{Ci}$ $\mathrm{L}-\left(\mathrm{U}-{ }^{14} \mathrm{C}\right)$ tyrosine (The Radiochemical Centre, Amersham) via peripheral ear vein during $6 \mathrm{~h}$ as described by Nicolas et al. (1977). Samples of the m. soleus were taken for analysis.

\section{Sample preparation and SDS gel filtration}

The sample preparation and the SDS gel filtration were described in a methodical paper (Schreurs et al., 1983). The most relevant details will be recapitulated. The preparation of the sample is adapted to the factors relevant to the formation of SDS protein complexes (Fish, 1975).

The final sample solution contains, among other components: protein $(2.5 \%)$, SDS $(6 \%)$ and 2-mercaptoethanol (2\%). In analytical runs the sample has been applied in a five-fold dilution.

Via a 1-ml sample loop the sample solution is aplied to a column $(90 \times 2.6 \mathrm{~cm})$ of Sephacryl S-400 Superfine (Pharmacia, Sweden) and eluted in the presence of SDS. The eluent of the column $(24 \mathrm{ml} / \mathrm{h})$ is monitored at $280 \mathrm{~nm}$ and collected in 200-drop $(\simeq 5 \mathrm{ml})$ fractions. These are analysed for ${ }^{14} \mathrm{C}$ radioactivity and total protein (Lowry et al., 1951).

\section{The (relative) specific activity}

The specific activity (SA) is expressed as the amount of radioactivity incorporated

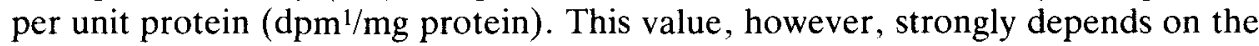
amount of radioactivity present in the precursor pool. For comparative purposes it is therefore more convenient to use the relative specific activity (RSA). The RSA of a protein fraction (f) is its specific activity $\left(\mathrm{SA}_{\mathrm{f}}\right)$ expressed relative to the specific activity of the total protein in the original sample $\left(\mathrm{SA}_{\mathrm{o}}\right)$. Thus $\mathrm{RSA}_{\mathrm{f}}=\left(\mathrm{SA}_{\mathrm{f}} / \mathrm{SA}_{\mathrm{o}}\right)$.

\section{Results and discussion}

An elution profile, typical for SDS solubilized proteins of the $\mathrm{m}$. soleus, monitored at $280 \mathrm{~nm}$, is shown in Fig. 1a. It has to be mentioned that due to the treatment with SDS and 2-mercaptoethanol only single-chain proteins and protein subunits can be recognized. Moreover, not only the proteins but practically all muscle constituents are present in the sample solution. For that reason the total elution profile consists

${ }^{1} \mathrm{dpm}$ : disintegrations per minute; $1 \mathrm{dpm} \approx 16.667 \mathrm{mBq}(\mathrm{Bq}$, becquerel, is the SI unit of radioactivity). 


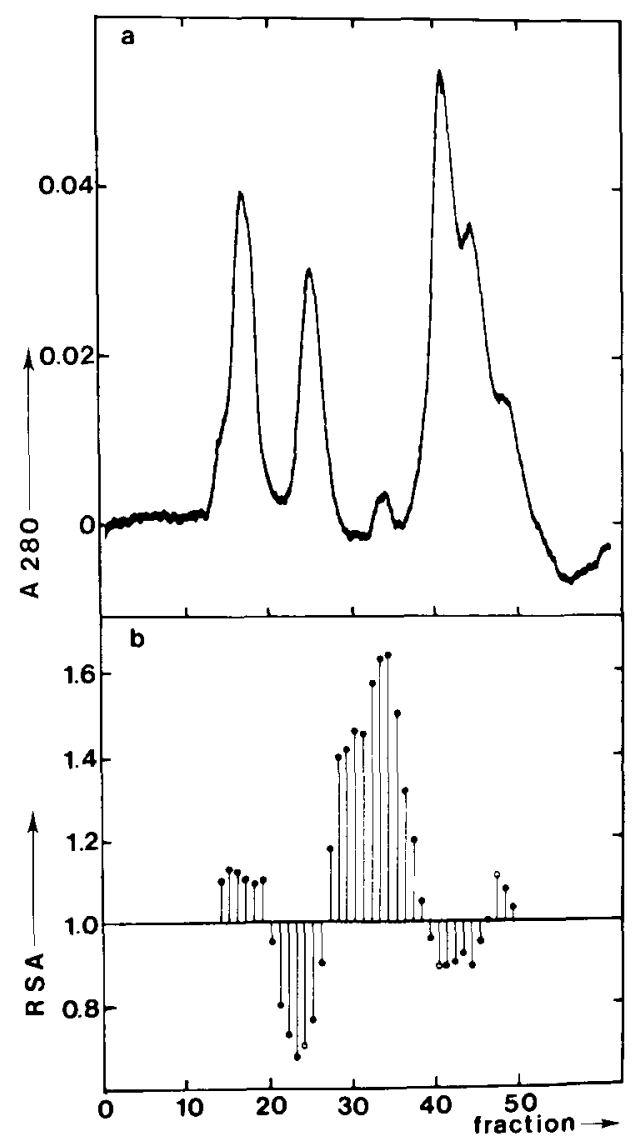

Fig. 1. a. Typical elution profile (protein part) of a m. soleus (rabbit), solubilized in SDS, on Sephacryl S400 Superfine monitored at $280 \mathrm{~nm}$ (analytical run). b. RSA (relative specific activity) spectrum of column fractions. $\mathrm{RSA}_{\mathrm{f}}=\left(\mathrm{SA}_{\mathrm{f}} / \mathrm{SA}_{\mathrm{o}}\right)$. mean values of six preparative runs.

of a protein part (Fig. 1a) and a non-protein part, which is not shown (Schreurs et al., 1983).

The RSA spectrum corresponding to the protein part (Fig. 1b) shows the distribution of the incorporated ${ }^{14} \mathrm{C}$-tyrosine over the protein components in the various column fractions. Therefore the RSA value of a column fraction may be considered as a first impression of the synthesis rate of the constituent proteins relative to the mean synthesis rate of total muscle protein. Information about individual proteins can be obtained if they can be located in one of the fractions.

As an example we have calculated the relative synthesis rates for actin and the myosin subunits. The fractions in which these polypeptides can be expected are roughly derived from the selectivity curve of the column (Schreurs et al., 1983) on account of their molecular weight (Waterlow et al., 1978). The purest fractions are 


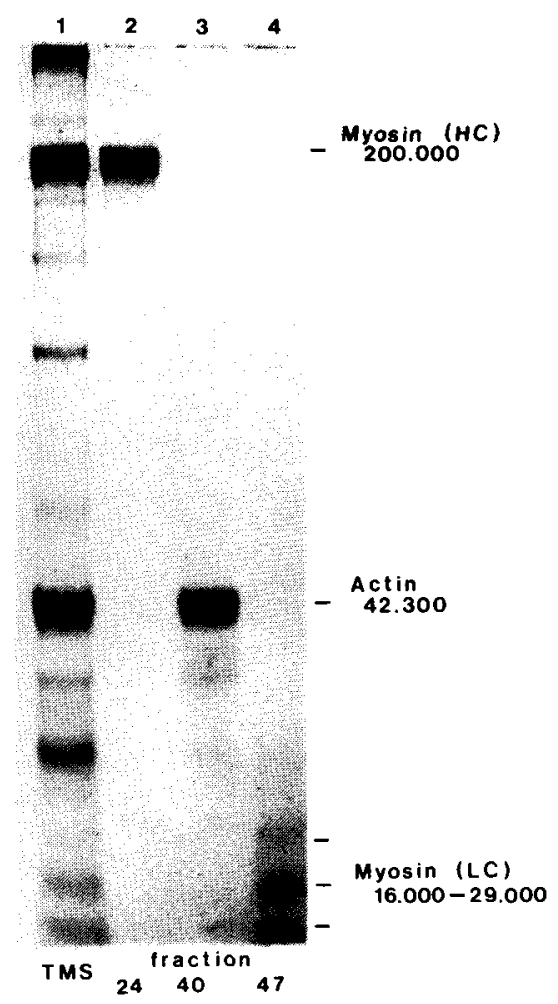

Fig. 2. Electrophoretic purity of preparative column fractions. $1=$ total muscle sample (TMS); $2=$ fraction 24 , myosin heavy chains (MHC); 3 = fraction 40 , actin (ACT); 4 = fraction 47 , myosin light chains (MLC).

selected by SDS gel electrophoresis and are shown in Fig. 2. The calculated results are presented in Table 1.

Decisive to our approach is the ultimate purity of the proteins in the fractions. In comparison with other approaches this aspect has to be weighted against the change in specific activity of the proteins, a severe and unpredictable complication in most isolation procedures (Lobley \& Lovie, 1979). The results of SDS gel electrophoresis are, however, encouraging. In the present example the myosin heavy chains are practically pure in fraction 24 . The same holds for the myosin light chains in fraction 47 , if they are considered as a group. The purity of actin may be problematical. This protein is supposed to occur in the largest elution peak. This peak, however, will also accommodate some other proteins e.g. the subunits of the myofibrillar troponin and tropomyosin and the sarcoplasmic enzymes enolase, aldolase and creatine kinase. Nevertheless the protein of fraction 40 , the top fraction of this peak, has a high electrophoretic purity.

Our results, indicating a non-uniform synthesis rate for the myofibrillar proteins, agree with most data in literature as reviewed by Millward (1980). There is, howev- 
Table 1. Relative turnover rates of the main muscle proteins.

\begin{tabular}{llllll}
\hline Polypeptide/Subunit & MW & $\begin{array}{l}\text { Purest } \\
\text { fraction }\end{array}$ & $\begin{array}{l}\text { RSA }^{1} \\
(n=6)\end{array}$ & Tyr $^{2}$ & $\begin{array}{l}\text { Relative turnover } \\
\text { rate }^{3}\end{array}$ \\
Myosin heavy chains & 200000 & 24 & $0.70 \pm 0.05$ & 2.2 & 1.5 \\
Myosin light chains & $16000-2900047$ & $1.11 \pm 0.14$ & 1.6 & 3.3 \\
Actin & 42300 & 40 & $0.89 \pm 0.06$ & 4.3 & 1.0 \\
\hline
\end{tabular}

$1 \mathrm{RSA}=$ relative specific activity

2 Corrections for differences in tyrosine content (percentage of amino acid residues) have to be made. Published amino acid compositions of myosin (Koizumi, 1974; Lowey \& Holt, 1973) and actin (Collins \& Elzinga, 1970) have been used.

3 Relative turnover rate calculated as the amount of ${ }^{14} \mathrm{C}$-tyrosine per unit tyrosine. The values are expressed relative to actin.

er, a lack of unanimity about the relative values of the synthesis rates for the individual proteins. Only Lobley \& Lovie (1979) think in terms of a uniform synthesis rate. Obviously the heterogenity in synthesis rate does not only exist between proteins of thin and thick filaments (actin and myosin) but also between subunits of a single functional protein (light and heavy chains of myosin).

Thus, in accordance with results of other isolation procedures this new approach supports the conclusion that even functionally related proteins can show considerable differences in synthesis rate. These results support the suggestion that a functional unit, such as the myofibril, does not turnover as a whole. Therefore the ultimate renewal of a myofybril seems to be effected by replacing individual proteins (or their subunits) at individual rates rather than by replacing the whole unit at one time.

Our approach of estimating the relative synthesis rates of individual proteins is an obvious alternative for methods with low protein recovery (sometimes less than $25 \%$ ). If considerable amounts of protein get lost it cannot be excluded that the specific activity of the proteins changes during their isolation. A change in specific activity of proteins will appear with an unequal loss of labelled and unlabelled material. This is most likely to occur in the myofibril, where newly synthesized proteins at first loosely attach to the outside of this organel (Morkin, 1970). As in many experiments less than a few per cent of all protein is labelled, a non-uniform distribution of the labelled proteins may interfere with the isolation procedure and as a consequence give rise to conflicting results. Such effects do not influence our results since the present approach considers the total amount of labelled and non-labelled protein material. Therefore we consider our data, derived from the RSA spectrum, as fairly accurate.

The differences in RSA values observed for the myofibrillar proteins can, in our opinion, not be explained by a contamination of sarcoplasmic proteins, which are supposed to have relative high turnover rates (Lebherz et al., 1982). Although such a contamination may have some influence on the observed values it is unable to explain the observed differences. The protein fractions under consideration all have a high electrophoretic purity.

From the RSA spectrum it can be derived if and in which direction a certain RSA 
value will be influenced by contaminating proteins. The myosin heavy chains are surrounded by proteins with a higher RSA value. However, the purity and abundance of the subunits in the top fraction (24) of the second elution peak support the accuracy of the calculated value. In the case of actin possible positive and negative effects seem to counterbalance. At the left hand side the RSA values are considerably higher but the protein contamination is low. At the right hand side the RSA values are lower but the contamination is somewhat larger. The myosin light chains, if considered as a group, seem to be uncontaminated. An equal turnover rate for the various polypeptides should require RSA values in the same ratio as their tyrosine contents. Our results provide no evidence at all for such values.

Another advantage of our approach is the additional information obtained about the protein metabolism of the muscle under consideration. This can be useful in comparative studies. The elution profile gives information about the differences in the protein populations of various muscles e.g. the nature and relative abundance of individual proteins. The RSA spectrum, in addition, gives an overall impression of their relative synthesis rates. This is especially useful in comparative studies about the influence of physiological parameters on the synthesis rate of individual muscle proteins. Moreover the required information can be obtained by a simple and straightforward procedure which handles all proteins in the same way.

\section{References}

Collins, J. H. \& M. Elzinga, 1975. The primary structure of actin from rabbit skeletal muscle. Journal of Biological Chemistry 250:5915-5920.

Fish, W. W., 1975. Determination of the molecular weight of membrane proteins and polypeptides. In: E. D. Korn (Ed.), Methods in membrane biology, Vol. 4, p. 189-276. New York, Academic Press.

Koizumi, T., 1974. Turnover rates of structural proteins of rabbit skeletal muscle. Journal of Biochemistry 76: 431-439.

Lebherz, H. G., J. K. Tetell, J. E. Shackelford \& M. J. Sardo, 1982. Regulation of concentrations of glycolytic enzymes and creatine phosphate kinase in fast twitch and slow twitch skeletal muscles of the chicken. Archives of Biochemistry and Biophysics 214: 642-656.

Lobley, G. E. \& J. M. Lovie, 1979. The synthesis of myosin, actin and the major protein fractions in rabbit. Biochemical Journal 182: 867-874.

Lowey, S. \& J. C. Holt, 1973. An immunochemical approach to the interaction of light and heavy chains in myosin. Cold Spring Harbor Symposia on Quantitative Biology 37: 19-28.

Lowry, O. H., N. J. Rosebrough, A. L. Farr \& R. J. Randall, 1951. Protein measurement with the Folin phenol reagent. Journal of Biological Chemistry 193: 265-275.

Millward, D. J., 1980. Protein degradation in muscle and liver. Comprehensive Biochemistry. 19B (I): 153-232.

Morkin, E., 1970. Postnatal muscle fiber assembly: Localization of newly synthesized myofibrillar proteins. Science 167: 1499-1501.

Nicholas, G. A., G. E. Lobley \& C. I. Harris, 1977. Use of the constant infusion technique for measuring rates of protein synthesis in the New Zealand White rabbit. British Journal of Nutrition 38: 1-17.

Schreurs, V. V. A. M., H. A. Boekholt \& R. E. Koopmanschap, 1983. Gel filtration in sodium dodecyl sulphate of hydrophobic muscle proteins on Sephacryl S-400 Superfine. Journal of Chromatography 254: 203-210.

Waterlow, J. C., P. J. Garlick \& D. J. Millward, 1978. Protein turnover in mammalian tissues and in the whole body. Amsterdam, Elsevier North Holland. 